\title{
Quantum phase slips and number-phase duality in disordered TiN nanostrips
}

\author{
I. Schneider, ${ }^{1}$ K. Kronfeldner, ${ }^{1}$ T. I. Baturina, ${ }^{1,2,3}$ and C. Strunk ${ }^{1, *}$ \\ ${ }^{1}$ Institute of Experimental and Applied Physics, University of Regensburg, D-93040 Regensburg, Germany \\ ${ }^{2}$ Institute of Semiconductor Physics, 13 Lavrentjev Avenue, Novosibirsk 630090, Russia \\ ${ }^{3}$ Novosibirsk State University, Pirogova Str. 2, Novosibirsk 630090, Russia
}

(Received 25 September 2017; revised manuscript received 13 March 2019; published 29 March 2019)

\begin{abstract}
We have measured the electric transport properties of TiN nanostrips with different widths. At zero magnetic field, the temperature-dependent resistance $R(T)$ saturates at a finite resistance toward low temperatures, which results from quantum phase slips in the narrower strips. We find that the current-voltage $(I-V)$ characteristics of the narrowest strips are equivalent to those of small Josephson junctions. Applying a transverse magnetic field drives the devices into a reentrant insulating phase, with $I-V$ characteristics dual to those in the superconducting regime. The results provide evidence that our critically disordered superconducting nanostrips behave like small self-organized random Josephson networks.
\end{abstract}

DOI: 10.1103/PhysRevB.99.094522

\section{INTRODUCTION}

The loss of global phase coherence due to quantum phase slips (QPSs) is a prime element in the understanding of resistive or even insulating states in one-dimensional (1D) superconducting systems [1-3]. In single Josephson junctions [4,5] and 1D Josephson junction arrays [6], phase slippage is meanwhile rather well understood. In small junctions, the Josephson coupling competes with Coulomb charging effects, leading to an uncertainty relation between the phase and Cooper pair number and thus QPS. If the Josephson coupling energy $E_{J}$ is comparable to or smaller than the charging energy $E_{C}$, supercurrent is suppressed by QPSs that persist down to zero temperature. QPSs can be either incoherent or coherent. The former are dissipative and lead to a finite resistance, whereas the latter give rise to the Coulomb blockade of Cooper pair tunneling, i.e., strongly insulating behavior below a critical voltage $V_{c}$. Above $V_{c}$, a typical back-bending ("Bloch nose") of the $I-V$ characteristics is observed [4] that indicates Bloch oscillations of the voltage. These are the electrodynamic dual to the current oscillations of the standard ac Josephson effect [7]. Achieving this dual to the Josephson effect in a circuit which can support a dc electric current is of fundamental interest for quantum metrology.

The situation is less clear for homogeneously disordered nanowires. At higher temperatures, the phase slip rate, and thus the resistance, behaves thermally activated. In the zero temperature limit, it either vanishes exponentially or saturates at a finite value by virtue of quantum tunneling. When the superconducting state in the wire is spatially homogeneous, the phase slips are delocalized along the wire, as opposed to an inhomogeneous superconductor, where local phase slips occur preferentially at weak spots. To avoid inhomogeneities due to grain boundaries, the wires are often made of amorphous materials like $\mathrm{Mo}_{\mathrm{x}} \mathrm{Ge}_{1-\mathrm{x}}, \mathrm{Nb}_{\mathrm{x}} \mathrm{Si}_{1-\mathrm{x}}$, or Ti [8-12]. Coherent

*christoph-.strunk@physik.uni-regensburg.de
QPSs were recently demonstrated in amorphous $\operatorname{InO}_{x}[13]$ and $\mathrm{NbN}$-wires [14].

For critically disordered films, i.e., films close to the disorder-induced superconductor-insulator transition (SIT), it has been suggested that the superconducting condensate is intrinsically fractionalized into small droplets [15-17]. This prediction is meanwhile supported by accumulating experimental evidence in ultrathin films [18-22]. The experiments show that random fluctuations of the superconducting gap occur on a typical scale of $20-50 \mathrm{~nm}$, which is larger than the superconducting coherence length $\xi \simeq 9 \mathrm{~nm}$. In TiN and $\mathrm{NbN}$, the gap fluctuations are uncorrelated with the underlying fine-crystalline structure of the metal film.

Hence, the question arises how the disorder induced gap fluctuations manifest themself in narrow strips, when the strip width becomes comparable to the typical length scale of the fluctuations. We expect that the transport properties of the strips deviate from those of both 2D films and homogeneous nanowires in that random weak spots start to dominate their behavior. If indeed Josephson-coupled [23] granules are formed, the supercurrent will be dominated by the smallest Josephson coupling energy $E_{J}$ in the system. When $E_{J}$ is sufficiently small, charging effects will reduce the maximal Josephson current via quantum fluctuations of the phase. In addition, we anticipate that the magnetic flux enclosed between adjacent granules will reduce the dominating Josephson coupling energy with respect to the charging energy $E_{C}$, resulting in Coulomb blockade and strongly insulating behavior.

Here, we address the above questions in a systematic study of the properties of nanostrips of width $8 \xi<w<50 \xi$ made of a critically disordered ultrathin superconducting TiN film of thickness $d<\xi$. We find a gradual suppression of the zero-resistance state with a low temperature resistance saturating at a value that increases as the strips narrow down. The current-voltage characteristics strongly resemble those of small Josephson junctions for the narrowest strips. At finite magnetic field, we observe reentrant behavior and highly insulating states; the $I-V$ characteristics being dual to those 
observed in the superconducting state in that current and voltage are interchanged. The insulating state is characterized by a critical voltage that is modulated by the magnetic field. Our results provide evidence that superconducting strips made from critically disordered films behave as random Josephson networks.

\section{SAMPLES AND MEASUREMENT CIRCUITRY}

The 780-nm-long nanostrips are prepared by subtractive patterning from a TiN film with a thickness of $3.6 \mathrm{~nm}$ and an initial sheet resistance of $2.5 \mathrm{k} \Omega$ at $300 \mathrm{~K}$ [24]. Across two deposited $\mathrm{Au} / \mathrm{Ti}$ electrodes with a thickness of $120 \mathrm{~nm}$, a strip-shaped area of PMMA (polymethyl methacrylate) is exposed to an electron beam at a very high dose $\left(30 \mathrm{mC} / \mathrm{cm}^{2}\right)$ that creates cross-links between the polymer strands and renders the exposed areas insoluble. The noncross-linked PMMA around these areas is then removed in an acetone bath. In a last step, the TiN strip is defined by argon ion etching. After processing, the sheet resistance $R_{\square}$ of the wider strips increased to $4.3 \mathrm{k} \Omega$ at $300 \mathrm{~K}$. Because of the deformation of the cross-linked resist during etching, the width of the narrow strips was hard to measure accurately. Most consistent results for the width were obtained from the ratio $R / R_{\square}$ at $300 \mathrm{~K}$. For a design width of $100 \mathrm{~nm}$, the width estimated from $R / R_{\square}$ deviated typically by $20 \%$.

We voltage bias the sample to measure the dc current with a Femto transimpedance amplifier DDPCA-300-S with integrated biasing circuit. Pi filters at room temperature and two stages of copper powder filters at $\mathrm{mK}$ temperatures provide an efficient high frequency filtering. In some measurements, we have added two $50 \mathrm{k} \Omega$ resistors at the chip carrier in series. The bias resistors shift the load line of the device and also provide access to the usually hysteretic part of the $I-V$ characteristics. All resistance values were extracted from the linear regime of the full $I-V$ characteristics (see below and Appendix B).

\section{SUPERCONDUCTING STATE}

\section{A. Temperature dependence of resistance}

Figure 1 shows the linear resistance of several strips vs temperature $T$. While $R(T)$ approaches zero at low temperatures in the widest strips, it clearly saturates for the narrower ones. Such behavior is in line with earlier observations [3,12]. The saturation can be explained by a quantum saturation of the phase slip rate at the lowest temperatures.

To analyze the temperature dependence of the resistivity at higher temperatures, we have fitted the data in Fig. 1 using a simplified version of the Langer-Ambegaokar model [25-27],

$$
R(T)=R_{0} \exp \left(-\Delta F_{0}(T) / k_{\mathrm{B}} T\right),
$$

where $R_{0}$ is a fixed prefactor, and the activation energy corresponds to the loss of superconducting condensation energy near the mean-field transition temperature, $T_{c}$,

$$
\Delta F_{0}(T)=k_{\mathrm{B}} T_{0} \times\left(1-T / T_{c}\right)^{3 / 2} .
$$

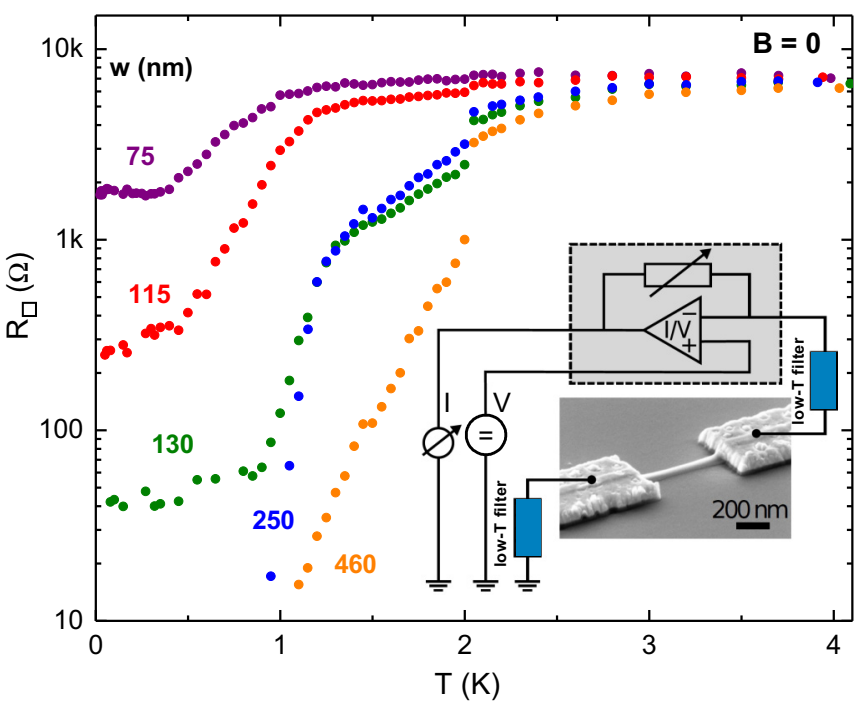

FIG. 1. Sheet resistance, $R_{\square}$, vs temperature, $T$, at zero magnetic field of TiN nanostrips of length $780 \mathrm{~nm}$ and with different widths, $w$. Inset: Scanning electron micrograph of a typical sample and sketch of the measurement setup.

The characteristic temperature, $T_{0}$, of the activation process is given by

$$
T_{0}=\frac{\xi_{\mathrm{GL}} w t}{k_{\mathrm{B}} \mu_{0}}\left(\frac{\hbar}{4 e \kappa \xi_{\mathrm{GL}}^{2}}\right)^{2},
$$

which is controlled near the $T_{c}$ by the Ginzburg-Landau coherence length $\xi(T)=\xi_{\mathrm{GL}} /\left(1-T / T_{c}\right)^{1 / 2}$ and the GinzburgLandau parameter $\kappa$. The regime of thermally activated resistance is clearly visible in the Arrhenius plot, in Fig. 2(a), while the resistance of the narrower strips saturates at gradually increasing resistance levels. Solid lines are fits corresponding to Eq. (1). The activation temperatures extracted from these fits are shown in Fig. 2(b). The prefactor $R_{0} \simeq 6.7 \pm 1 \mathrm{k} \Omega$
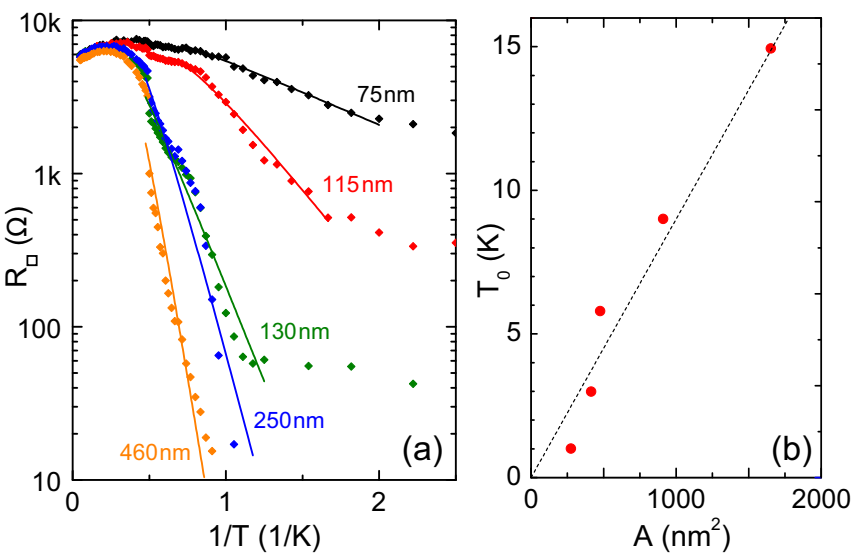

FIG. 2. (a) Arrhenius plot of the resistance at zero magnetic field for different strip width of chip A. The lines are fits according to Eq. (1). The resistance of the narrowest strips saturates at low temperature. The lines are fits according to Eq. (1). (b) Activation temperatures $T_{0}$ vs cross section $A=w t$ extracted from the fits in (a). The slope of the dashed line corresponds to an effective GinzburgLandau parameter of $\kappa_{\text {eff }} \simeq 490$. 


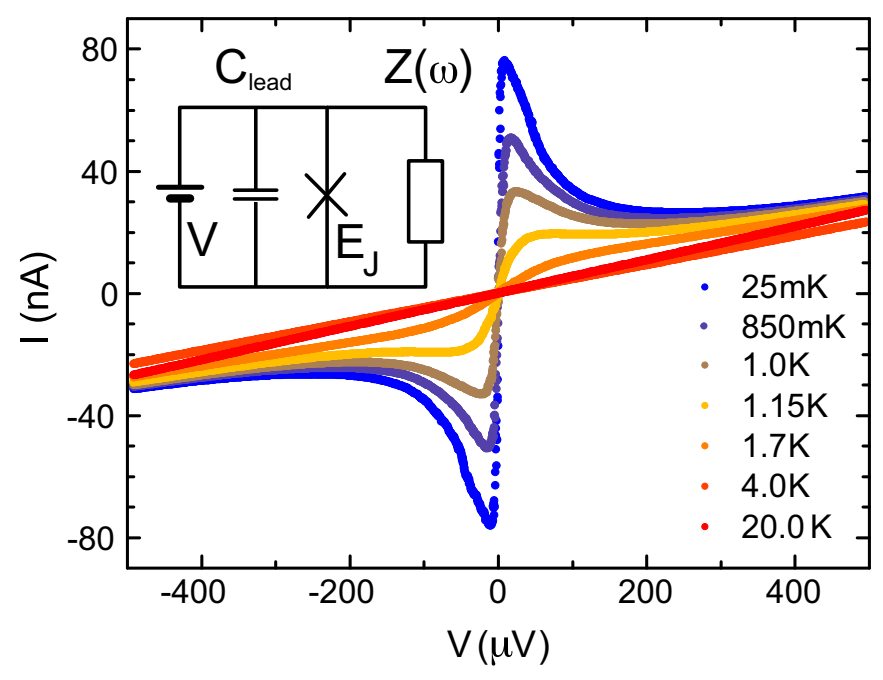

FIG. 3. Temperature evolution of $I-V$ characteristics for a 250-nm-wide strip. Inset: Voltage source and parallel connection of the active phase slip element and the environmental impedance $Z(\omega)$. At zero frequency $\operatorname{Re}[Z(\omega=0)]=R_{N}$ corresponds to the slopes of $I(V)$. The lead capacitance renders the parallel and the serial connection equivalent at high frequencies where $\operatorname{Re}\left[Z\left(\omega=\omega_{p}\right)\right]=$ $R_{B}$, the bias resistor in the IZ theory (see text).

slightly scatters in a range of $2 \mathrm{k} \Omega$ around the quantum resistance $R_{Q}=6.45 \mathrm{k} \Omega$. We observe a good proportionality between the values of $T_{0}$ and the strip width, $w$, as expected from Eq. (3).

Taking $\xi_{\mathrm{GL}}=8.9 \mathrm{~nm}$ [24], we can extract an effective Ginzburg-Landau-parameter of $\kappa_{\text {eff }} \simeq 490$ from the slope of the interpolation line. This value is a factor 2.5 larger than the $\kappa$ value obtained independently on the same TiN-film [24]. Given the fact that our strips are considerably wider than the coherence length and very close to the SIT, such agreement is very satisfactory. It suggests that the concept of thermally activated phase slips (in our case, more phase slip lines [28]) can at least qualitatively be transferred to films substantially wider than the coherence length [29].

\section{B. Supercurrent peak in narrow wires}

Figure 3 presents the evolution of $I-V$ characteristics with temperature for a device with $w=250 \mathrm{~nm}$. As a function of voltage bias, we observe a relatively narrow supercurrent peak at low bias. At low voltages, the behavior strikingly resembles the observations of small tunneling junctions [30]. In tunnel junctions, the current rapidly drops to zero at higher bias after the supercurrent peak and remains zero up to $V=2 \Delta$. In our devices, the current does not drop to zero, but is gradually taken over by a parallel conduction channel with a resistance $R_{N}$ that is close to the normal state resistance of the strips [31]. Similar observations are made for the even narrower strips shown in Fig. 4. With decreasing strip width, the supercurrent peak is gradually suppressed. In contrast, the $I-V$ characteristics of the wider strips feature a much more complex nonequilibrium behavior with several different regimes that also is found in large 2D films (see Appendix B) [32].

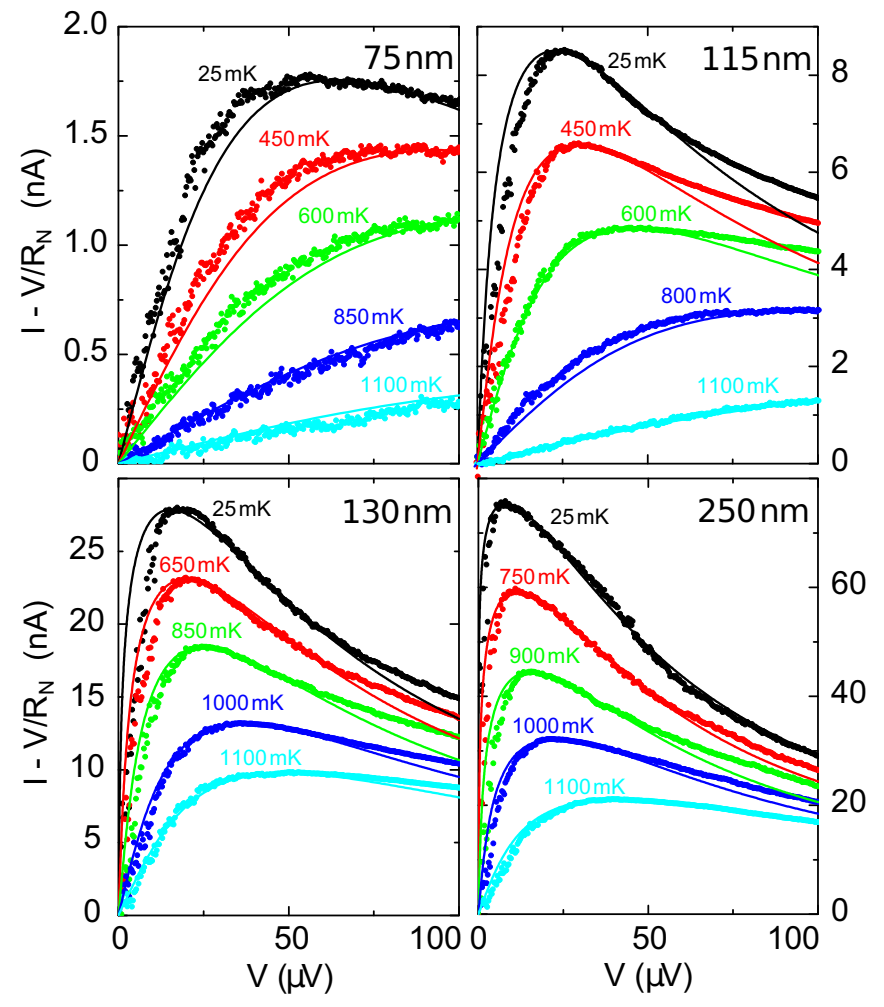

FIG. 4. Supercurrent peaks of the four narrowest wires on chip A. The solid lines are fits according to the IvanchenkoZilberman model with $V=V_{\mathrm{IZ}}-R_{B} I$ (see text).

Small tunneling junctions are well described by the Ivanchenko-Zilberman (IZ) theory, assuming that the voltage bias is applied using a frequency-independent resistor $R_{B}$ in series to the junction $[33,34]$. To understand our results, an additional frequency-dependent impedance $Z(\omega)$ parallel to the junction has to be taken into account. At low frequencies, the real part of this impedance is given essentially by the normal state resistance: $\operatorname{Re}[Z(\omega=0)]=R_{N}=20.6 \mathrm{k} \Omega$ for the device presented in Fig. 3.

After subtraction of the current contribution $V / R_{N}$ from the measured $I-V$ characteristics (see Fig. 4), we compare our experimental results to an adaption of the IZ model, in which the smearing of the critical current with increasing temperature is caused by the Johnson-Nyquist noise of a resistor $R_{B}$ that is used to voltage bias the Josephson junction. The current-voltage characteristics in this model reads

$$
I\left(V_{\mathrm{IZ}}\right)=I_{0} \Im \mathrm{m}\left[\frac{\mathcal{J}_{1-\hbar V_{\mathrm{IZ}} / 2 e R_{B} k_{B} T}\left(\hbar I_{0} / 2 e k_{B} T\right)}{\mathcal{J}_{-\hbar V_{\mathrm{IZ}} / 2 e R_{B} k_{B} T}\left(\hbar I_{0} / 2 e k_{B} T\right)}\right],
$$

where $\mathcal{J}_{v}(z)$ is the modified Bessel function, $V_{\mathrm{IZ}}=V+R_{B} I$ being the voltage of the junction and the bias resistor $R_{B}$ connected in series, while $V$ denotes the measured voltage across the junction only. In our experiment, the role of $R_{B}$ is taken over by $\operatorname{Re}\left[Z\left(\omega_{p}\right)\right]$, the real part of the environmental impedance near the plasma frequency $\omega_{p}$ of the junction (see below).

Figure 4 shows a comparison of the theory with the supercurrent peaks observed in the four narrowest devices on chip A. The maximal supercurrent differs by more than a 
(a)

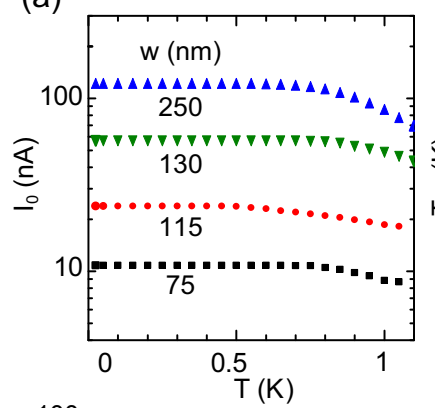

(b)

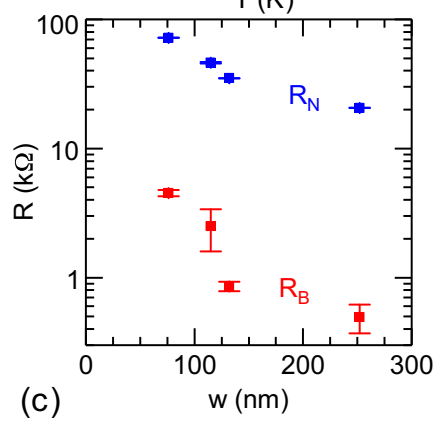

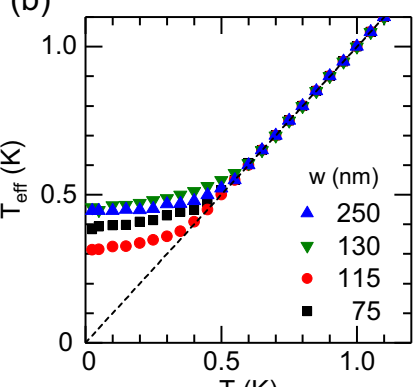

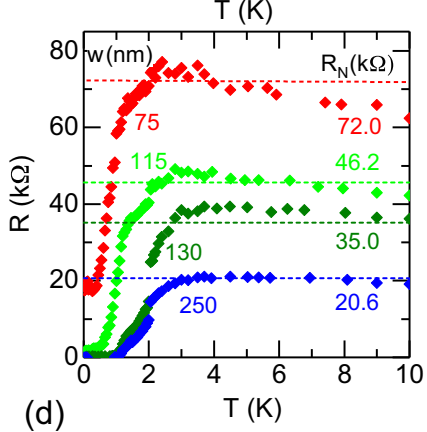

(d)

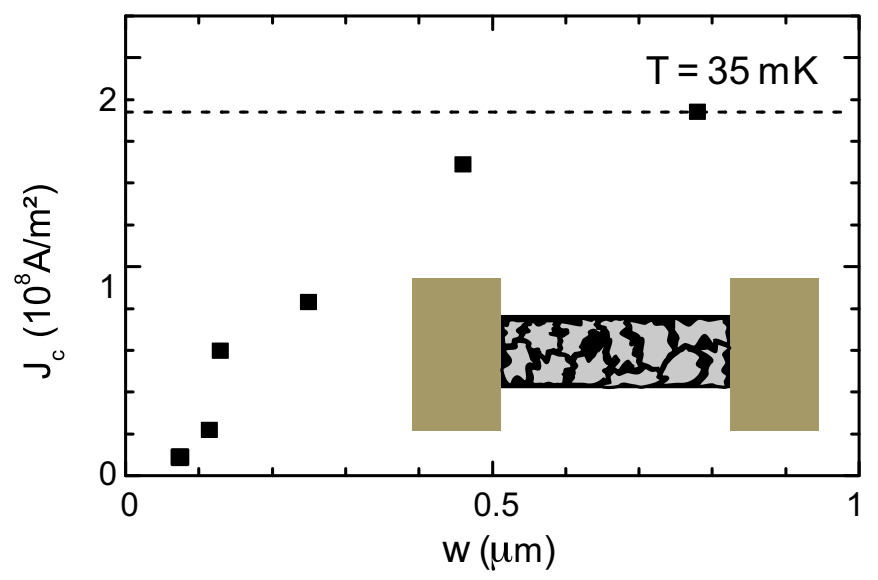

FIG. 6. Critical current density $J_{c}$ extracted from the maximal supercurrent vs nanostrip width. The dashed line indicates the expectation for a standard superconductor with $J_{c}$ independent of the strip width. Inset: Schematic of droplet structure of the superconducting order parameter.

curves for all four samples in Fig. 4. The $R_{B}$ values mainly affect the decay of the supercurrent above the maximum. We find $R_{B}<R_{N}$ as expected for an electromagnetic environment with shunting lead capacitances.

The critical current density $J_{c}$ extracted from the fitted $I-V$ characteristics is plotted vs strip width in Fig. $6 . I_{0}$ decreases above $600 \mathrm{mK}$, consistent with a decrease of the energy gap $\triangle$ above $T_{C} / 2$.

A very similar overproportional reduction of the switching current $I_{\mathrm{sw}} / I_{\mathrm{sw}, \max }$ was observed in chains of small Josephson junctions with decreasing ratio $E_{J} / E_{C}[6]$.

\section{INSULATING STATE}

charging energy. The phase fluctuations lead to a saturation of the height and width of the supercurrent peak, which is exactly what we observe below $\simeq 450-750 \mathrm{mK}$, depending on the device. The supercurrent peaks of all four devices can be fitted within the IZ theory using Eq. (4), if we model the effect of a finite charging energy $E_{C}<E_{J}$ by replacing the bath temperature $T$ with an effective temperature $T_{\text {eff }}$. The intrinsic critical current $I_{0}$ and $T_{\text {eff }}$ together determine the height of the supercurrent peak. For a single $I(V)$ trace, the fit values of $T_{\text {eff }}$ and $I_{c 0}$ are dependent on each other. This ambiguity is removed by the demand that $I_{c 0}$ should first increase with decreasing $T_{\text {bath }}$ and then saturate, while $T_{\text {eff }}$ should first be proportional to the measured temperature $T$, then deviate from $T$ and eventually saturate at the lowest $T$.

The resulting values of $I_{c 0}$ and $T_{\text {eff }}$ are shown in Figs. 5(a) and 5(b), respectively. Given our low temperature filtering, the fit values extracted for $T_{\text {eff }}$ at low $T$ are far too large to be explained by artifacts like residual noise. The saturation of $T_{\text {eff }}(w)$ correlates with the saturation of $R(T \rightarrow 0, w)$ in Fig. 1 and is very likely a manifestion of QPS, i.e., small but, finite values of $E_{C} / E_{J}$ not captured by the IZ theory.

The values of $R_{N}$ determined independently from the high voltage limit $I-V$ characteristics are plotted vs strip width in Fig. 5(c) (blue squares) together with those for $R_{B}$ (red squares). As illustrated in Fig. 5(d), the values of $R_{N}$ extracted from the fits correlate very well with the maxima of the $R(T)$

\section{A. Superconductor-insulator transition}

Next we turn to the behavior in the magnetic field. The purple curve in Fig. 7 shows the $I-V$ characteristics of a nanostrip (labeled A) with $w=85 \mathrm{~nm}[R(4 \mathrm{~K}) \simeq 72 \mathrm{k} \Omega]$ at $B=0$. The superconducting transition is rounded, with the maximum of $I$ missing and the linear resistance $R(T \rightarrow 0) \simeq$ $4 \mathrm{k} \Omega$ remaining finite, similar to the narrower nanostrips in Fig. 1 . The Josephson-like $I-V$ characteristics at $B=0$ can be converted into a strongly insulating one in a perpendicular magnetic field $B=2.4 \mathrm{~T}$ (orange curve). The linear resistance $R(T)$ of the device is plotted for different magnetic fields in the upper left inset. In addition, we show a similar evolution of $R(T)$ of another nanostrip (labeled B) prepared in a different run with $w=75 \mathrm{~nm}$ (see Fig. 1) in the lower right inset. As seen in both insets to Fig. 7, the resistance initially decreases as superconducting correlations develop in an intermediate temperature regime $(0.3-1 \mathrm{~K})$. At lower temperatures, a reentrant insulating behavior is observed for both devices. At the lowest temperatures, the linear resistance can exceed the normal state resistance by three orders of magnitude.

The degree of insulation varies strongly between different devices, and also between different cool-downs of the same device. Although highly insulating states appear predominantly in the narrower nanostrips, the behavior is not 


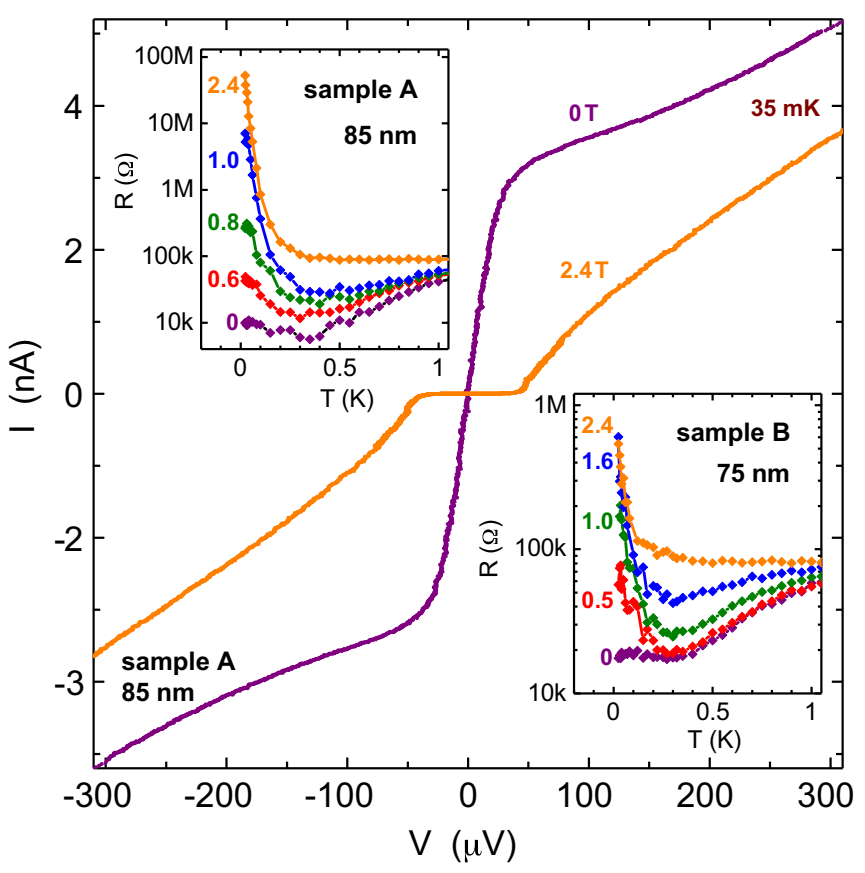

FIG. 7. $I-V$ characteristics of nanostrip A $(w=85 \mathrm{~nm})$ for $B=0$ (purple) and in perpendicular magnetic field $B=2.4 \mathrm{~T}$ (orange). Two $50 \mathrm{k} \Omega$-resistors were placed adjacent to the device to access the back-bending part of the $I-V$ characteristics. Insets: $R(T)$ for nanostrips $\mathrm{A}$ and $\mathrm{B}(w=75 \mathrm{~nm})$ with an inititially superconducting and reentrant behavior with increasing magnetic field and for nanostrip A very high values of the linear resistance.

solely controlled by the nanostrip width, but also depends on fluctuations of the disorder potential that determines the spatial distribution of the superconducting-order parameter in a sample specific way. The strongly insulating behavior in our narrowest nanostrips $(w<100 \mathrm{~nm})$ in the magnetic field is distinct from that of amorphous $\mathrm{InO}_{\mathrm{x}}$ nanowires [37]. In the insulating state, the resistance of the latter saturates at comparatively low values.

\section{B. Insulation at intermediate magnetic fields}

In Fig. 8(a), we show the evolution of the $I-V$ characteristics of nanostrip A with increasing temperature at $B=2 \mathrm{~T}$. A cold bias resistor allows us to access also the back-bending parts of the $I-V$ characteristics. The voltage drop over the serial impedance $I / G_{N}$ (which is dual to the contribution $V / R_{N}$ of a parallel impedance to the current in the inset to Fig. 3) is subtracted from the measured bias voltage. At low $T$, a pronounced Bloch nose [7] develops in the $I-V$ characteristics, which is gradually suppressed at higher $T$. The maximal voltages $V_{c}(T)$ at the Bloch nose are the dual counterparts of the current maxima $I_{c}(T)$ in Fig. $4 ; V_{c}(T)$ is quickly suppressed with increasing temperature.

Next, we demonstrate that the behavior in the reentrant insulating regime at finite magnetic field $0.5 \mathrm{~T} \lesssim B \lesssim 2.4 \mathrm{~T}$ obeys a strong duality symmetry when compared to the zero field case. A necessary prerequisite for a strong current blockade by QPSs is the isolation of the active elements by a high impedance environment. The environmental impedance can
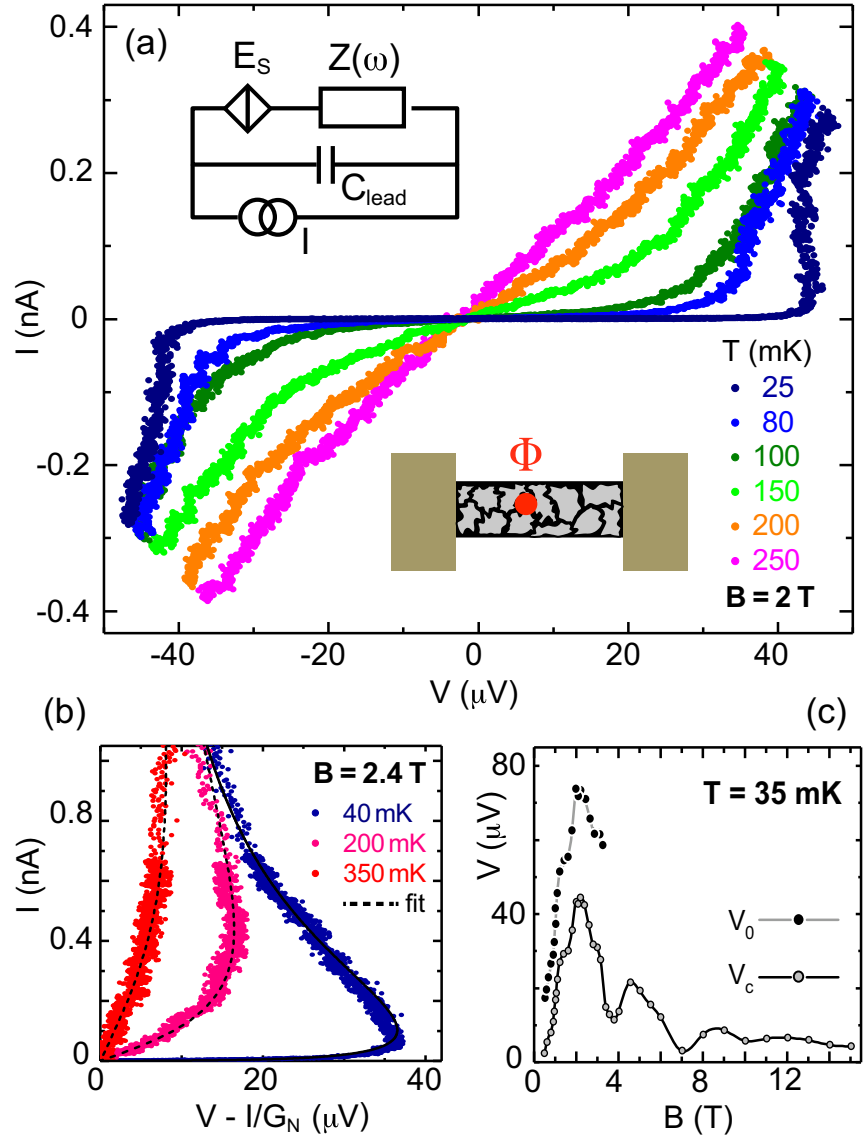

FIG. 8. (a) Evolution of $I-V$ characteristics of nanostrip A ( $w=$ $85 \mathrm{~nm}$ ) with $T$ at $B=2 \mathrm{~T}$ in the insulating regime. Upper inset: Dual measurement circuit with a serial connection of the active phase slip element and the environmental impedance $Z(\omega)$. Lower inset: Droplet structure of superconducting order parameter with magnetic flux induced weakest spot (red). (b) Fits with the dual IZ theory after subtraction of $I / G_{N}$ from the measured voltage. (c) Magnetic field dependence of the critical voltages $V_{c}$ and $V_{0}$ (see text).

be either resistive $[10,11,38]$ or inductive $[1,4,5,39]$. The onchip leads toward our nanostrips are made from low-resistive gold. Hence, the only candiates for a high-impedance element protecting the active spot of the nanostrips are the more robust sections of the strip adjacent to the active spot. In the superconducting state, we estimate the kinetic inductance (using $R_{N}=67 \mathrm{k} \Omega$ and $T_{c} \simeq 1.5 \mathrm{~K}$ ) to be $L_{\text {kin }} \simeq 0.14 \hbar R_{N} / k_{B} T_{c} \simeq$ $380 \mathrm{nH}$. With the gradual suppression of superconducting correlations in high magnetic field, the large normal state resistance $R_{N}$ of the nanostrip gradually takes over.

To understand the $I-V$ characteristics also in the insulating regime, we employ the dual version of the IZ model in the form [4]

$$
V\left(I_{\mathrm{IZ}}\right)=V_{0} \Im \mathrm{m}\left[\frac{\mathcal{J}_{1-e I_{\mathrm{IZ}} / \pi G_{B} k_{B} T}\left(e V_{0} / \pi k_{B} T\right)}{\mathcal{J}_{-e I_{\mathrm{IZ}} / \pi G_{B} k_{B} T}\left(e V_{0} / \pi k_{B} T\right)}\right],
$$

where $I_{\mathrm{IZ}}=I+G_{B} V$ is the current of the junction connected in parallel to the bias conductor $G_{B}=1 / R_{B}$, while $I$ denotes the current through the junction that is measured in our experiment. 
(a)

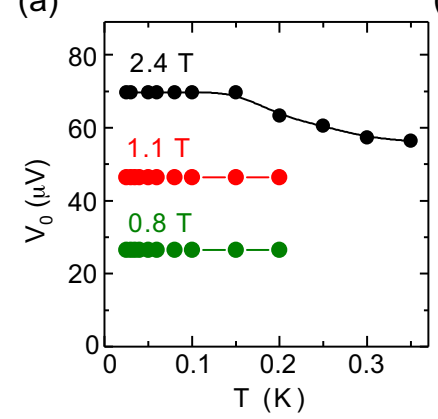

(b)
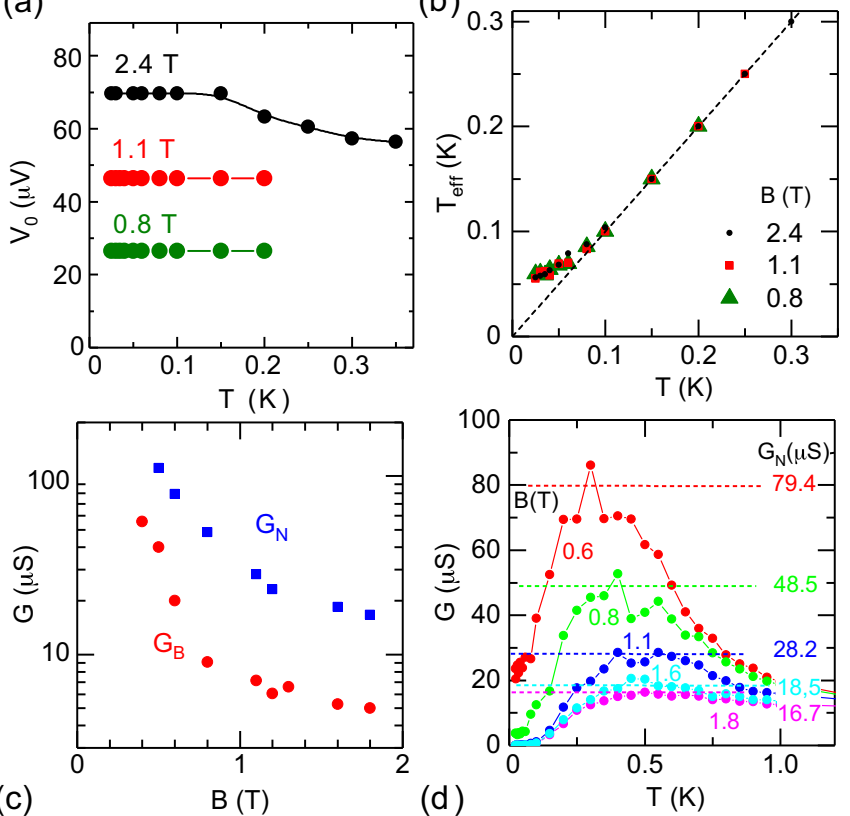

FIG. 9. (a) Critical voltage $V_{0}$ vs $T$ for several magnetic fields as extracted from the IZ fits (see text). (b) Effective temperature used in the dual IZ analysis of the Bloch nose in the reentrant insulating regime. (c) Corresponding fitparameters $G_{N}$ and $G_{B}$ extracted from the dual IZ analysis. (d) Conductance $G(T)$ in the insulating reentrance observed for the $85-\mathrm{nm}$-wide strip. The dashed horizontal lines denote the $G_{N}$ values from (c).

The good agreement between the data and the fits in Fig. 8(b) is evidence of the striking duality between the superconducting and insulating sides of the transition. The intrinsic critical voltage $V_{0}$ is extracted from the dual IZ fit and it exceeds $V_{c}(T=35 \mathrm{mK})$ by a factor two. It is dual to the intrinsic critical current $I_{0}(T)$ in Fig. 5(a). As a function of magnetic field, the critical voltage $V_{c}(B)$ plotted in Fig. 8(c) displays a strong peak centered around $B \simeq 2 \mathrm{~T}$ that was also observed for the switching voltages in $2 \mathrm{D}$ TiN films [18]. Note, however, that the strongly insulating behavior does not occur in the wider strips (see Appendix A). Hence, the insulation in large films must be of different origin, possibly involving long-range interactions [40,41]. Superimposed on the peak, an oscillatory behavior is observed that is also reflected in the magnetoconductance (see subsection $\mathrm{C}$ below). At higher magnetic fields, i.e., above $3 \mathrm{~T}$ (the upper critical field of less disordered TiN-films), it is still possible to simulate the measured $I-V$ characteristics using the IZ model. Because of the fractionalization of the condensate wave function, it is not obvious up to which magnetic field the superconductivity persists locally.

The temperature dependence of $V_{0}$ is displayed for different magnetic fields in Fig. 9(a). In contrast to $V_{c}(T)$ (not shown), the intrinsic critical voltage $V_{0}(T)$ remains essentially $T$ independent up to $200 \mathrm{mK}(150 \mathrm{mK}$ for $B=2.4 \mathrm{~T})$. As on the superconducting side, we introduce an effective temperature to include the effects of a finite Josephson coupling $E_{J}<E_{C}$ and residual noise in the system. An analogous fitting procedure results in values of $V_{0}$ and $T_{\text {eff }}$ that are shown in Figs. 9(a) and 9(b), respectively. When compared to their counterpart at the superconducting side [Fig. 5(b)] the values of $T_{\text {eff }}$ are an order of magnitude smaller and do not vary significantly with magnetic field.

Dual to the superconducting side, the environmental admittance $Z^{-1}(\omega)$ is now in series with the nanostrip. Similar to the superconducting case at $B=0$, the zero frequency limit $Z^{-1}(\omega=0)=G_{N}$ is reflected as a linear contribution $I / G_{N}$ to the $V(I)$ characteristics, which has to be subtracted from the measured voltage. The values of $G_{N}$ obtained from the high voltage part of the $V(I)$ characteristics are plotted vs magnetic field (blue squares) in Fig. 9(c). $G_{N}$ does not include the contribution from the two bias resistors $(2 \times 50 \mathrm{k} \Omega)$ mounted in series with and near to the sample chip. Analog to the superconducting side [compare to Fig. 5(d)], Fig. 9(d) shows that these values correlate very well with the maxima of the $G(T)$ curves for several values of the magnetic field.

At high frequencies, the lead capacitance again shorts the internal resistance of the voltage source. Hence, the parallel and serial connection of $Z(\omega)$ and the sample are equivalent (as on the superconducting side). Near the plasma frequency, $\operatorname{Re} Z^{-1}\left(\omega \simeq \omega_{p}\right)$ corresponds to the fit parameter $G_{B}$ in the dual IZ formula [Eq. (5)] that quantifies the smearing by thermal current noise: $G_{B}$ (red circles) controls the width of the Bloch nose [Fig. 8(b)]. In Fig. 9(c), we show the values of $G_{B}$ determined from the fits together with $G_{N}$ as a function of magnetic field. We find $G_{B}<G_{N}$ as expected from the duality to the superconducting side.

Also dual to the resistance $R(T)$ at the superconducting side [see Fig. 5(d)] the conductance $G(T)$ in the insulating regime-shown for different magnetic fields in Fig. 9(d)displays a maximum that shifts from $0.3 \mathrm{~K}$ to $0.5 \mathrm{~K}$ from the lowest to the highest field. All curves converge at higher temperatures where the magnetoresistance of the sample becomes small.

\section{Magnetoresistance in narrow strips}

Figure 10 displays the low temperature magnetoresistance of the wire with $w=85 \mathrm{~nm}$. Superimposed on a large peak, the magnetoresistance displays wiggles that are reminiscent to the magnetoconductance fingerprints familiar from disordered normal-metallic wires [42]. Such random fluctuations are consistent with fluctuations of the local $E_{J}$ in our disordered TiN nanostrips $(w<100 \mathrm{~nm})$. They are expected to cause complex frustration patterns in a random multiloop topology.

Such multiloop topology can also explain the observed fluctuation pattern of $V_{c}(B)$ (see Fig. 8(c), as the magnetoresistance at the weakest spot is controlled by the combination of a few Josephson loops with random sizes. The characteristic resistances $R_{\mathrm{N}}$ and $R_{\mathrm{B}}$ extracted from the $I-V$ characteristics are shown as blue and red squares, respectively. As seen in the inset of Fig. 10, both $R_{\mathrm{N}}$ and $R_{\mathrm{B}}$ gradually saturate in the limit of high magnetic field.

\section{DISCUSSION}

In our devices, we use the nanostrip width and the magnetic field to tune the phase slip rate $E_{S}$ over a wide range to span the whole transition from a fully superconducting 


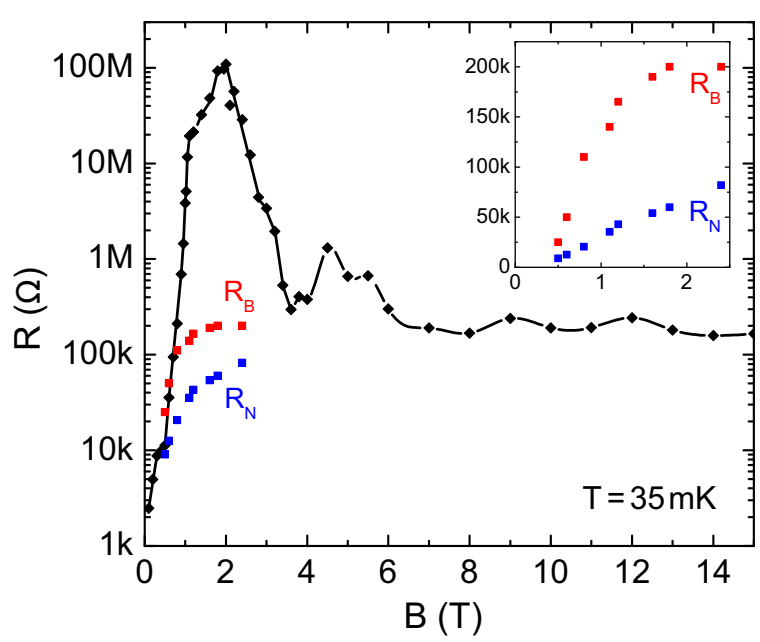

FIG. 10. Magnetoresistance of the wire with $w=85 \mathrm{~nm}$ (black diamonds). The resistances $R_{\mathrm{N}}=1 / G_{\mathrm{N}}$ extracted from the high voltage part of the $I-V$ characteristics (blue squares) and $R_{\mathrm{B}}=1 / G_{\mathrm{B}}$ from the IZ fits (red squares) are shown. The inset shows the evolution of the fit parameters $R_{N}$ and ${ }_{B}$ (see last section).

toward a fully Coulomb blockaded nanostrip. The pronounced sensitivity to the magnetic field can be naturally explained in terms of emerging granularity, [15-22] because the local Josephson coupling energies are gradually suppressed by the magnetic flux enclosed between the granules. Another source for the sensitivity to magnetic field is the global suppression of the local order parameter by the field that is expected to induce a parity effect favoring single electron over Cooper pair tunneling above a certain magnetic field [43]. In zero magnetic field and $w=250 \mathrm{~nm}$, we find Josephson behavior with a resistance below our detection limit (Fig. 1). Reducing $w$ to $\simeq 75 \mathrm{~nm}$ induces noticable quantum phase fluctuations and a corresponding saturation of $R(T)$ (Fig. 1). The phase fluctuations are manifested further in a gradual suppression of the supercurrent peak (Fig. 7). The estimated critical current is ten times smaller than expected from the reduction of the nanostrip width.

In presence of random granularity, the behavior of the strip will be dominated by the weakest spot, where Josephson coupling between the droplets of the fractionalized condensate is smallest. As shown in Ref. [44], rather small variations of the normal state resistance along nominally homogeneously disordered nanowires can induce substantial variations of local QPS amplitude $E_{S}$. Differences between different wires are most pronounced in the magnetic field. With increasing magnetic field, quantum phase fluctuations at the weakest spot will develop into coherent QPSs. The corresponding increase of $E_{S}$ leads to the emergence of current blocking and eventually strongly insulating behavior. Such behavior has been demonstrated in individual Josephson junctions embedded into 1D Josephson arrays as high impedance leads [4,5]. Furthermore, the reentrant $R(T)$ curves observed in some of our TiN nanostrips (insets in Fig. 8) are indistinguishable from those obtained earlier on artificial 1D Josephson arrays [45].

From the maximal value of $E_{S}(B)=2 e V_{0}(B) / 2 \pi=$ $4 e^{2} / 2 C_{\text {eff }}(B)$ (see Refs. [46,47]) we estimate $E_{S}(2 \mathrm{~T}) \simeq$ $13 \mu \mathrm{eV}$ from Fig. 8(d) and infer an effective capacitance
$C_{\text {eff }}(2 \mathrm{~T}) \simeq 0.6 \mathrm{fF}$. This value is much larger than the estimated geometric capacitance $C_{\text {geo }} \simeq 1.5 \mathrm{aF}$ [48]. The reduction of $E_{S}(B)$ with respect to $4 e^{2} / 2 C_{\text {geo }}$ by the residual Josephson coupling is dual to the suppression of $I_{0}(w)$ (Fig. 6) on the superconducting side. The effective capacitance $C_{\text {eff }}$ has contributions both from the geometric capacitance $C_{\text {geo }}$ between the granules and the kinetic capacitance $C_{\mathrm{kin}}$ that arises from the hybridization between Coulomb parabolas with adjacent numbers of Cooper-pairs [7]. $C_{\mathrm{kin}}$ is determined by the inverse curvature of the 2e-periodic energy-charge relation $E(Q)$ (the dual of the energy-phase relation of conventional Josephson junctions) and diverges at the inflection points of $E(Q)$, similar to the divergence of the band mass in a periodic potential. In the insulating regime of our critically disordered superconducting nanostrips, we expect the kinetic capacitance to dominate over the geometric capacitance in a similar way, as the kinetic inductance dominates over the geometric inductance of our nanostrips at $B=0$.

\section{CONCLUSION}

A magnetic-field-induced SIT is observed in mesoscopic TiN nanostrips. The $I-V$ characteristics on both sides of the transition are well described by dual versions of the IZ model. We provide strong evidence that the insulating state is related to Coulomb blockade induced by coherent QPSs. Our observations are consistently explained in terms of a fractionalization of the superconducting condensate in nanostrips made of critically disordered TiN films.

\section{ACKNOWLEDGMENTS}

We thank M. Baklanov for the supply of TiN material, D. Haviland, Yu. Nazarov, A. Ustinov, A. Shnirman, A. Mirlin, and T. Duty for inspiring discussions. T.I.B. acknowledges support by the Alexander von Humboldt Foundation, by the Ministry of Education and Science of the Russian Federation, the Russian Science Foundation under Project No. 14-2200143, and from the Consejería de Educación, Cultura y Deporte (Comunidad de Madrid) through the talent attraction program, Ref. No. 2016-T3/IND-1839.

\section{APPENDIX A: EFFECT OF MAGNETIC FIELD IN WIDER STRIPS}

In Fig. 11, we show the effect of magnetic field on the $R(T)$ curves for some of the wider TiN strips both on semilogarithmic scales $\lg R$ vs $T$ (left column) and $R$ vs $\lg T$ (right column). The representation of $\lg R$ vs $T$ (left column) shows that for $w=460 \mathrm{~nm}$ the transition temperature is gradually suppressed, while the resistance goes to zero for $B \leqslant 2.6 \mathrm{~T}$. The resistance $R(T, B=3 \mathrm{~T}) \simeq R_{N} / 2$, indicates that the critical magnetic field of the film is close to $3 \mathrm{~T}$. For $w=250 \mathrm{~nm}$ and $B \gtrsim 0.4 \mathrm{~T}$, the magnetic field induces a saturation of $R(T)$ at low temperatures. The saturation level $R_{\text {sat }}$ gradually increases with the field. The increasing $R_{\mathrm{sat}}$ is again consistent with an increase of the QPS rate that results from a suppression of the Josephson coupling energy with increasing magnetic field. For $w=130 \mathrm{~nm}$, the saturation occurs in zero field already. 

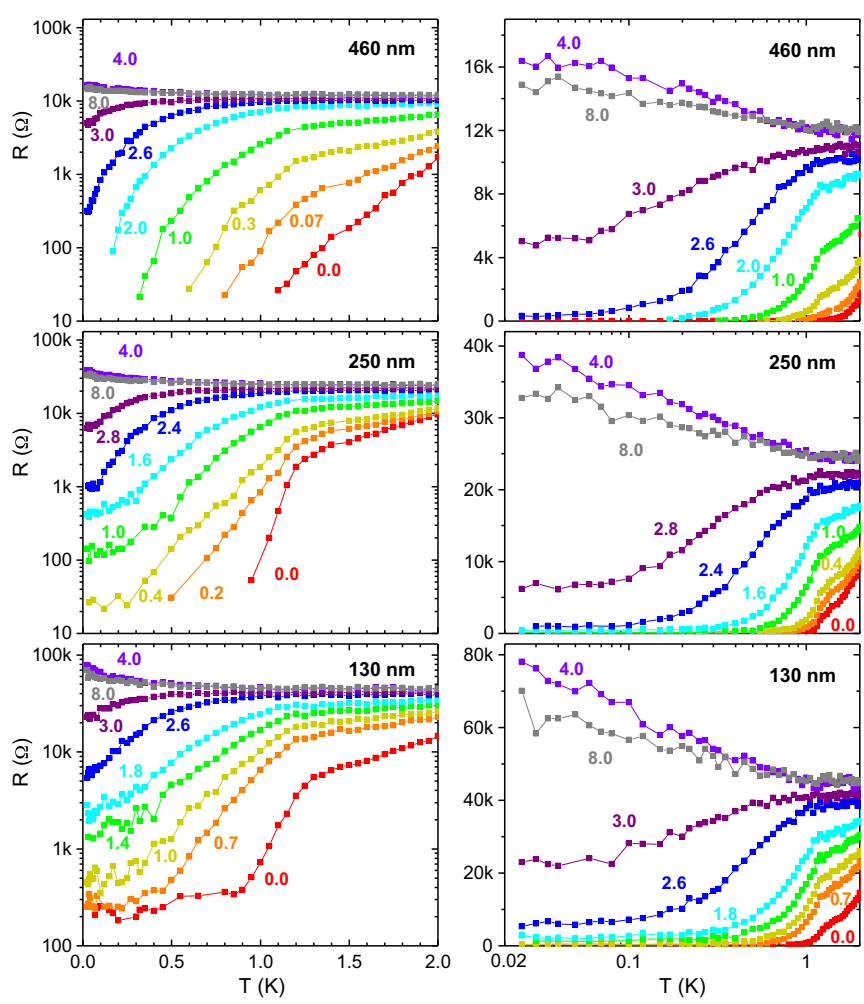

FIG. 11. Resistance vs temperature in perpendicular magnetic field for the wider TiN strips.

The representation of the resistance $R$ vs $\lg T$ (right column) shows that no strongly insulating reentrance is observed in these devices at any field. This observation supports our view that the reentrant behavior discussed in this paper requires the presence of a dominating weak spot of the sample, where a current blockade occurs that is induced by the proliferation of QPSs. At magnetic fields $B \gtrsim 4 \mathrm{~T}$, that exceeds the critical field of the TiN, $R(T)$ logarithmically rises as expected from the quantum corrections to the resistivity in a strongly disordered metal. For smaller nanostrip width $w<130 \mathrm{~nm}$, these corrections become quite substantial (80\%). It remains, however, much smaller than the resistance increase induced by the Coulomb blockade of Cooper pairs that appears responsible for the reentrant behavior in these devices (see Fig. 10 below). The absence of a highly insulating state in the wider TiN strips supports the idea that that the highly insulating states in 2D films of TiN (and possibly also $\mathrm{InO}_{x}$ ) are not local properties of these materials, but may result from long-ranged Coulomb interactions with a very large electric screening length [40,41].

\section{APPENDIX B: EVOLUTION OF I-V CHARACTERISTICS WITH DECREASING STRIP WIDTH}

In Fig. 12, we show the evolution of the $I-V$ characteristics with increasing wire width. For the narrowest strips, the supercurrent peak is very small and rounded. The slope of $I(V)$ remains finite, but decreases with the strip width. The temperature corresponding to the lowest slope of $I(V)$ in the high voltage regime, i.e., near the temperature of the maximum in $R(T)$ [see Fig. $5(\mathrm{~d})$ ] decreases from 4.5 to $2.8 \mathrm{~K}$ when $w$ decreases from 800 to $75 \mathrm{~nm}$. This leads to a crossing of the $I-V$ characteristics at high voltages for the narrower
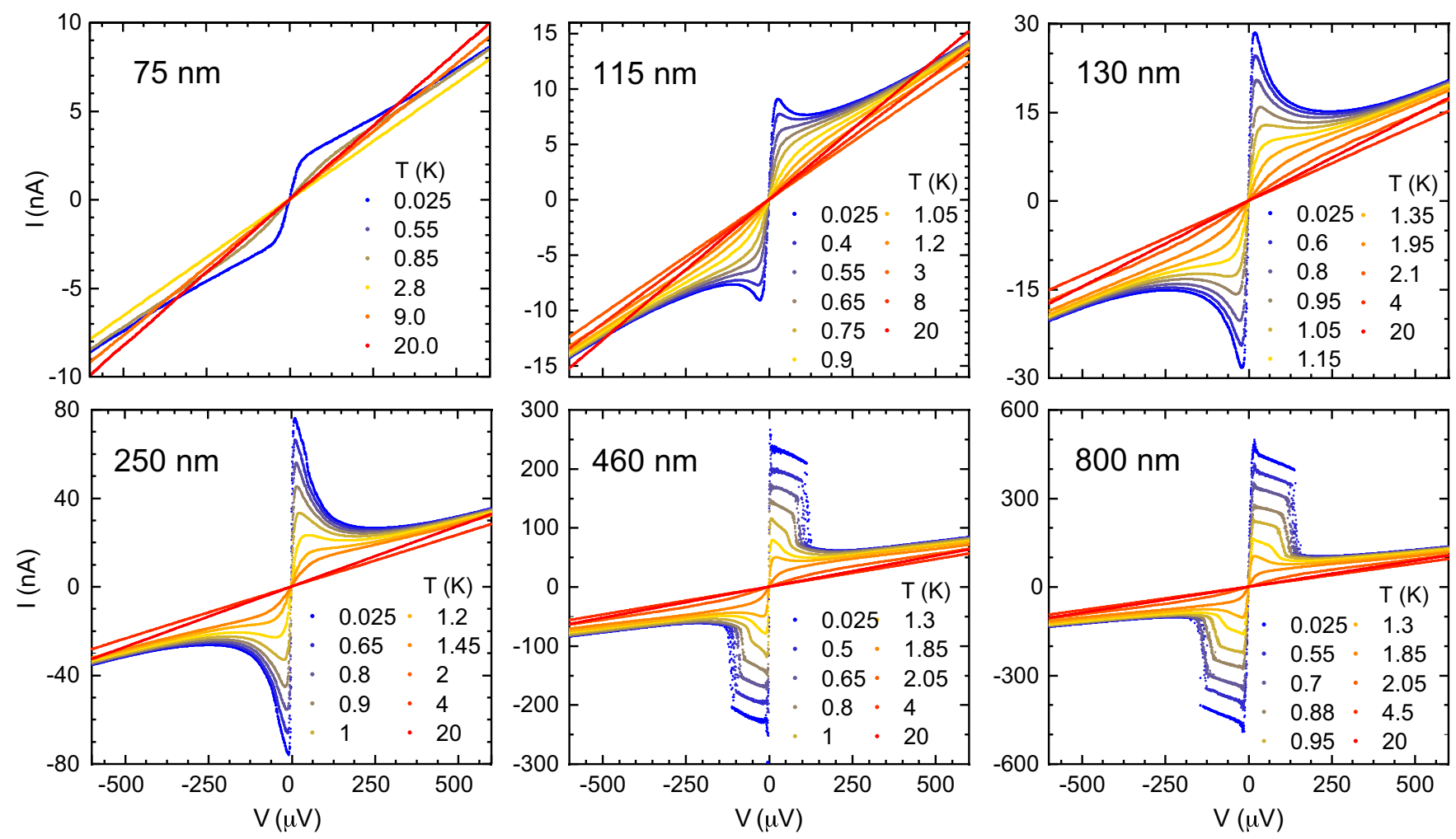

FIG. 12. $I-V$ characteristics for different nanostrips' widths and temperatures in zero magnetic field. 
wires. At a magnetic field of $5 \mathrm{~T}$, the differential conductance $d I(V) / d V$ of the narrower strips displays a zero bias anomaly, i.e., a narrow dip around zero bias, similar to observations in MoGe nanowires [49]. The amplitude of the dip corresponds to the resistance increase below $1 \mathrm{~K}$ at such magnetic field (right column in Fig. 11). These comparatively weak nonlinearities are consistent with the temperature dependence of the conductance, if electron heating occurs at elevated voltages. Electron heating is relevant, because the electron phonon coupling in metals becomes so weak at the lowest temperatures that the local electron temperature in the wire raises above the phonon temperature. The electric power required to significantly change the differential conductance amounts to a fraction of a picowatt- two orders of magnitude larger than the power near the current/voltage peaks in Figs. 2(a) and 4(a), respectively.

For the wider strips ( 480 and $800 \mathrm{~nm}$ ), the form of the $I-V$ characteristics changes drastically: After a very sharp peak, a shoulder forms in $I(V)$ at a rather high current level, which drops abruptly to a plateau at much lower current around 100$150 \mu \mathrm{V}$ and then gradually approaches the normal state resistance. The shoulder is identified as a "relaxation-oscillation" plateau known from conventional Josephson junctions [50]. It vanishes above $T \gtrsim 1 \mathrm{~K}$ and reflects dynamical oscillations of voltage and current that are controlled by the time constants of the $L C$ circuit formed by the sample together with its electromagnetic environment. The lower plateau is followed by gradual approach of $I(V)$ toward the normal state $I-V$ characteristics that is typical for the so-called hot spot behavior that is known from superconducting microbridges [51]. Such a combination of relaxation-oscillation and hot-spot behavior is also found for millimeter-sized 2D TiN films [32]. The crossover between the two types of $I-V$ characteristics constitutes further evidence that the character of the junctions dynamics drastically changes from microbridgelike toward Josephson-like as the strip widths is reduced.

The nonlinear resistance of the nanostrip (not shown) in a magnetic field of $5 \mathrm{~T}$ can be used to estimate the relevance of heating effects in the insulating regime. Similar to the data in Ref. [49], voltages around $100 \mu \mathrm{V}$ at $35 \mathrm{mK}$ are needed for an appreciable heating effect, corresponding to a current of $2 \mathrm{nA}$ and a power input of $0.2 \mathrm{nW}$. Near the Bloch nose in Fig. 4(a), the power dissipation is about $2 \mathrm{fW}$, and thus two orders of magnitude smaller. Hence, we can exclude heating effects as the origin of the nonmonotonicity in the $I-V$-curves in the strongly insulating regime.
[1] D. Haviland, K. Andersson, P. Ågren, J. Johansson, V. Schöllmann, and M. Watanabe, Physica C 352, 55 (2001).

[2] K. Y. Arutyunov, D. S. Golubev, and A. D. Zaikin, Phys. Rep. 464, 1 (2008).

[3] A. Bezryadin, J. Phys.: Condens. Matter 20, 043202 (2008).

[4] S. Corlevi, W. Guichard, F. W. J. Hekking, and D. B. Haviland, Phys. Rev. Lett. 97, 096802 (2006).

[5] A. Ergül, J. Lidmar, J. Johansson, Y. Azizoglu, D. Schaeffer, and D. B. Haviland, New J. Phys. 15, 095014 (2013).

[6] I. M. Pop, I. Protopopov, F. Lecocq, Z. Peng, B. Pannetier, O. Buisson, and W. Guichard, Nat. Phys. 6, 589 (2010).

[7] K. K. Likharev and A. B. Zorin, J. Low Temp. Phys. 59, 347 (1985).

[8] A. T. Bollinger, A. Rogachev, and A. Bezryadin, Europhys. Lett. 76, 505 (2006).

[9] A. T. Bollinger, R. C. Dinsmore, A. Rogachev, and A. Bezryadin, Phys. Rev. Lett. 101, 227003 (2008).

[10] C. H. Webster, J. C. Fenton, T. T. Hongisto, S. P. Giblin, A. B. Zorin, and P. A. Warburton, Phys. Rev. B 87, 144510 (2013).

[11] J. S. Lehtinen, K. Zakharov, and K. Y. Arutyunov, Phys. Rev. Lett. 109, 187001 (2012).

[12] J. S. Lehtinen, A. Kempinen, E. Mykkänen, M. Prunna, and A. J. Manninen, Supercond. Sci. Technol. 31, 015002 (2017).

[13] O. V. Astafiev, L. B. Ioffe, S. Kafanov, Y. A. Pashkin, K. Y. Arutyunov, D. Shahar, O. Cohen, and J. S. Tsai, Nature 484, 355 (2012).

[14] S. E. de Graaf, S. T. Skacel, T. Hönigl-Decrinis, R. Shaikhaidarov, H. Rotzinger, S. Linzen, M. Ziegler, U. Hübner, H. G. Meyer, V. Antonov, E. Il'ichev, A. V. Ustinov, A. Y. Tzalenchuk, and O. V. Astafiev, Nat. Phys. 14, 590 (2018).

[15] Z. Ovadyahu and D. Kowal, Solid Stats Commun. 90, 783 (1994).
[16] A. Ghosal, M. Randeria, and N. Trivedi, Phys. Rev. Lett. 81, 3940 (1998).

[17] A. Ghosal, M. Randeria, and N. Trivedi, Phys. Rev. B 65, 014501 (2001).

[18] T. I. Baturina, A. Y. Mironov, V. M. Vinokur, M. R. Baklanov, and C. Strunk, Phys. Rev. Lett. 99, 257003 (2007).

[19] B. Sacépé, C. Chapelier, T. I. Baturina, V. M. Vinokur, M. R. Baklanov, and M. Sanquer, Phys. Rev. Lett. 101, 157006 (2008).

[20] H. Q. Nguyen, S. M. Hollen, M. D. Stewart, J. Shainline, A. Yin, J. M. Xu, and J. M. Valles, Phys. Rev. Lett. 103, 157001 (2009).

[21] G. Lemarié, A. Kamlapure, D. Bucheli, L. Benfatto, J. Lorenzana, G. Seibold, S. C. Ganguli, P. Raychaudhuri, and C. Castellani, Phys. Rev. B 87, 184509 (2013).

[22] C. Carbillet, S. Caprara, M. Grilli, C. Brun, T. Cren, F. Debontridder, B. Vignolle, W. Tabis, D. Demaille, L. Largeau, K. Ilin, M. Siegel, D. Roditchev, and B. Leridon, Phys. Rev. B 93, 144509 (2016).

[23] Josephson coupling across weak links is not restricted to standard tunnel junctions. The presence of highly transmissive channels in the weak link typically leads to higher harmonics in the current-phase-relation.

[24] S. V. Postolova, A. Y. Mironov, and T. I. Baturina, JETP Lett. 100, 635 (2015).

[25] J. S. Langer and V. Ambegaokar, Phys. Rev. 164, 498 (1967).

[26] D. E. McCumber and B. I. Halperin, Phys. Rev. B 1, 1054 (1970).

[27] M. Tinkham, Introduction to Superconductivity, 2nd ed. (Dover Publications, Mineola, 1996).

[28] I. V. Zolochevski, Low Temp. Phys. 40, 867 (2014). 
[29] A. Y. Mironov, S. V. Postolova, and D. A. Nasimov, JETP Lett. 104, 766 (2016).

[30] A. Steinbach, P. Joyez, A. Cottet, D. Esteve, M. H. Devoret, M. E. Huber, and J. M. Martinis, Phys. Rev. Lett. 87, 137003 (2001).

[31] Such behavior is possible if the transmission probabilities $\tau$ between condensate droplets display a broad distribution between zero and one. For $\tau \gtrsim 0.1$, not only sequential tunneling, but also multiple Andreev reflections substantially contribute to the quasiparticle transport, which lead to a finite and approximately ohmic background conductance [M. Chauvin, P. vom Stein, D. Esteve, C. Urbina, J. C. Cuevas, and A. Levy-Yeyati, Phys. Rev. Lett. 99, 067008 (2007)].

[32] K. Kronfeldner et al. (unpublished).

[33] Y. M. Ivanchenko and L. A. Zil'berman, Zh. Eksp. Teor. Fiz. 55, 2395 (1968) [Sov. Phys. JETP 28, 1272 (1969)].

[34] V. Ambegaokar and B. I. Halperin, Phys. Rev. Lett. 22, 1364 (1969).

[35] G.-L. Ingold and H. Grabert, Phys. Rev. Lett. 83, 3721 (1999).

[36] J. Ankerhold, Europhys. Lett. 67, 280 (2004).

[37] S. Mitra, G. C. Tewari, D. Mahalu, and D. Shahar, Phys. Rev. B 93, 155408 (2016).

[38] T. T. Hongisto and A. B. Zorin, Phys. Rev. Lett. 108, 097001 (2012).
[39] D. B. Haviland, K. Andersson, and P. Ågren, J. Low Temp. Phys. 118, 733 (2000).

[40] T. I. Baturina and V. M. Vinokur, Ann. Phys. 331, 236 (2013).

[41] A. Y. Mironov, D. M. Silevitch, T. Proslier, S. V. Postolova, M. V. Burdastyh, A. K. Gutakovskii, T. F. Rosenbaum, V. V. Vinokur, and T. I. Baturina, Sci. Rep. 8, 4082 (2018).

[42] P. A. Lee and A. D. Stone, Phys. Rev. Lett. 55, 1622 (1985).

[43] T. Duty, K. Cedergren, S. Kafanov, R. Ackroyd, and J. H. Cole, arXiv: 1808.08552.

[44] M. Vanević and Y. V. Nazarov, Phys. Rev. Lett. 108, 187002 (2012).

[45] E. Chow, P. Delsing, and D. B. Haviland, Phys. Rev. Lett. 81, 204 (1998).

[46] J. E. Mooij and Y. V. Nazarov, Nat. Phys. 2, 169 (2006).

[47] J. E. Mooij, G. Schön, A. Shnirman, T. Fuse, C. J. P. M. Harmans, H. Rotzinger, and A. H. Verbruggen, New J. Phys. 17, 033006 (2015).

[48] As a rough estimate, we used $C_{\text {geo }} \simeq 8 \epsilon_{0} a$, the capacitance between infinity and a disk of radius $a \simeq 20 \mathrm{~nm}$. The capacitance between neighboring islands is usually smaller.

[49] H. J. Kim and A. Rogachev, Phys. Rev. B 94, 245436 (2016).

[50] F. L. Vernon and R. J. Pedersen, J. Appl. Phys. 39, 2661 (1968).

[51] W. J. Skocpol, M. R. Beasley, and M. Tinkham, J. Appl. Phys. 45, 4054 (1974). 